Nuclear Physics B89 (1975) 287-306

(c) North-Holland Publishing Company

\title{
EXTENSION OF THE CHOU-YANG MODEL TO MULTIPARTICLE PROCESSES *
}

\author{
F.S. HENYEY and U.P. SUKHATME \\ Randall Laboratory of Physics, University of Michigan, Ann Arbor, Michigan 48104
}

Received 18 November 1974

\begin{abstract}
A natural and essentially unique extension of the Chou-Yang model to multiparticle processes is presented. The multiparticle amplitudes are derived from the physical ideas of the original Chou-Yang approach. The beam and target particles are treated as extended, non-recoiling, Lorentz-contracted objects which interact and produce particles as they pass through each other. The resulting multiparticle amplitudes satisfy $s$-channel unitarity exactly.

A minor modification allows us to include diffractive inelastic effects. When diffraction is included the connection with the electromagnetic form factor must be given up. The model is in good qualitative agreement with various features exhibited by the data. The model requires the total cross section to rise in a definite way. However, quantitative agreement at ISR energies is far from satisfactory. This is probably due to the "no-recoil" assumption inherent in the Chou-Yang model.
\end{abstract}

\section{Introduction}

An important constraint on models for multiparticle amplitudes is that they yield correct elastic and inelastic diffraction scattering results via unitarity calculations. The multiperipheral model faces extremely serious difficulties in this respect [1]. This leads us to consider alternative models for multiparticle production. The Chou-Yang model is an attractive suggestion for describing elastic scattering [2]. In this article, we extend the model to multiparticle production in a natural and essentially unique way. These multiparticle amplitudes, when used to calculate elastic scattering, exactly give the original Chou-Yang result.

The essential features of the Chou-Yang model are:

(i) Particles are treated as extended objects, and their scattering is determined from the matter overlap.

(ii) In calculating the matter overlap, an integration is performed parallel to the direction of motion, so that every piece of the incident particle, as it passes through the target particle, interacts with every piece of the target at the same transverse position no matter what the relationship between the longitudinal positions are. This

\footnotetext{
* Research supported in part by the US Atomic Energy Commission
} 
can be seen to be a "no recoil" approximation, since the amplitude for interaction is independent of whether the same piece of the incident or target particle has undergone previous interactions. This approximation is in contrast to that of, for example, the multiperipheral model, in which an interaction at some transverse position enhances the probability of subsequent nearby interactions.

(iii) The matter distribution used in calculating the overlap is the same as the charge distribution, which gives the electromagnetic form factor. As we shall see, this equality of matter and charge distributions, cannot hold because of the presence of inelastic diffraction processes. These processes can be handled in an analogous " $q$-number" model, also developed by Chou and Yang [3].

(iv) Eikonalization is included in the Chou-Yang model. This makes it possible for us to write down a multiparticle extension which incorporates unitarity.

(v) In its original form, there was no energy dependence in the model. However energy dependence has more recently been included [4], in an ad-hoc way. We shall see that our approach leads to definite predictions on the energy dependence.

Our extension is based on the ideas proposed by Chou and Yang. It appears to be the essentially unique application of these ideas to multiparticle processes. We take the no-recoil assumption as basic, and assume that even such fundamental recoil effects as energy-momentum conservation do not seriously perturb the results obtained by neglecting recoil. As each bit of the incident particle passes through the target particle, it can cause an interaction at a particular point in space and time. This interaction leads to the production or possibly to the destruction, of some object, which ultimately decays (if it is not subsequently destroyed in another interaction) into the observed particles. We refer to this object as a "cluster". (We will see later that a cluster must have vacuum quantum numbers so that, for example, it cannot be a single pion.)

The probability for such an interaction is given by the product of the matter densities of incident and target particles. Thus, the interaction amplitude $\rho(x, y, z, t)$ is the product of wave functions whose squares are the matter densities. One would like to identify this matter density with the charge density if possible. Since Chou and Yang assume that there is only one type of matter density, our parallel assumption is that there is only one type of cluster. This produced state in general is not an eigenstate of the mass operator. The bits of the incident and target particles that led to the interaction are not allowed to recoil, nor are they allowed to be enhanced or suppressed. In other words, the source $\rho$ is not affected by interactions. This property is the defining property of a classical source, one which can be specified independently of the quantum uncertainties connected with the presence of an interaction. Thus we see that the no-recoil assumption of Chou and Yang forces us to assume a classical source whose strength is the square root of the Chou-Yang matter overlap.

Because of the no recoil approximation, energy-momentum conservation cannot be exactly satisfied. The approximation would be more justifiable if the leading particles carried almost all of the incident energy. 
For the same reason, that we don't allow recoil, we cannot allow the produced clusters to interact among themselves. If we did, it would introduce a correlation absent in the Chou-Yang model. Thus the clusters are treated as free particles, except for their interaction with the classical source and their decay which is assumed to occur a long time after the collision is concluded, so that it does not affect the collision.

It is fortunate for us that, as is well known, the classical source problem has an exact solution and that the exact unitary $S$ matrix can be written down. We are able to calculate any quantity of interest. For example, our elastic scattering agrees exactly with that of Chou and Yang.

To finish the specification of the model, the wave function of each incoming particle is given by a spherically symmetric function $f(r)$ in that particle's rest frame. Lorentz transformations are done to transform to the same frame for both particles; for convenience we work in the center of mass frame. In order to make the total amount of matter be energy independent in spite of the Lorentz contractions, it is required that the wave functions be vectors. From phenomenological consideration we choose the simple form that the wave function $\psi_{\mu}$ have only a 4 th component in the particle's rest frame.

The only freedom in the model is the choice of the function $f(r)$, the choice of a coupling constant determining the strength of the interaaction, and the decay distribution of the clusters. The first two of these freedoms are exactly the same as for Chou and Yang. Our energy dependence comes completely from the Lorentz transformations.

Chou and Yang considered a further model in which the interactions can cause transitions to additional states of the same quantum numbers as the incoming particles [3]. We can do likewise by considering the wave functions to be matrices in the space of such states. This " $q$-number" model is to be preferred to the simpler one because it includes diffractive inelastic processes as well as elastic scattering.

The model we arrive at turns out to be the same as a model considered by Calucci, Jengo and Rebbi [5], as well as being a special case of a class of models considered by Aviv, Sugar and Blankenbecler [6]. The new features of our work, not discussed in ref. [5] are (i) the derivation of the model from the Chou-Yang model and the implications of our results for the Chou Yang model, and (ii) the identification of the most important phenomenological aspects of the model.

A detailed description of our model and derivation of observable consequences is given in sect. 2 . The casual reader may wish to skip to sect. 3 , where a summary of the important qualitative results is presented. Sect. 4 contains a quantitative comparison of the extended Chou-Yang model with data, concentrating on the relationship between the growth of total cross section (and associated elastic slope) and the growth of the average multiplicity. Our conclusions are presented in sect. 5 . 


\section{2 . Description of the model}

In this section we present our formalism. The results used in subsequent sections are eqs. (10), (21), (29) and (25) for the "c-number model" and eqs. (42), (43) and (45) for the " $q$-number" model which includes diffractive inelastic scattering. The extreme asymptotic behaviors of the cross section and the multiplicity are given in eqs. (32)-(35). The inclusive cross section (in the central region) is given by eq. (37), with a particular example given by eq. (38).

As discussed in the introduction, we extend the Chou-Yang model to production processes by considering these processes to be described in terms of a classical source for production, and this source to be given by an overlap of wave functions of the two incident particles, which we refer to as "protons". The source density which we denote by $\rho$ is

$$
\rho(x, y, z, t)=g \psi_{1}(x, y, z, t) \psi_{2}(x, y, z, t),
$$

where we have introduced a coupling constant $g$ to provide the normalization. The wave functions $\psi_{1}$ and $\psi_{2}$ depend on the preparation of the incoming states and on the structure of the protons. Rather than using the experimentally feasible momentum eigenstates, we imagine the protons as approaching each other with center of mass energy $w=\sqrt{s}$, and at a relative impact parameter $\boldsymbol{b}$. To make experimental statements we must transform between $b$ and $t$. We work in COM coordinates, with the $z$-axis along the direction of motion of the protons, and the origin of coordinates half way between the protons (the $z$-axis bisects the impact parameter vector $b$ ); $t=0$ is chosen to be the time at which the center of each proton is at $z=0$. We define a transverse position vector $\beta=(x, y)$. In the rest frame of each proton, its wave function is spherically symmetric, being given by $\psi_{i}=f_{i}\left(R^{2}\right)$ where $R$ is the distance to its center. In the COM system each proton is Lorentz contracted [7]. Since we are interested in high energy collisions, we make the approximations

$$
\begin{aligned}
& v=1, \\
& s=4 M^{2} \gamma^{2},
\end{aligned}
$$

where $M$ is the proton rest mass. The Lorentz transformations give us the source

$$
\rho(\boldsymbol{\beta}, z, t)=g f_{1}\left(\left(\boldsymbol{\beta}-\frac{1}{2} \boldsymbol{b}\right)^{2}+\gamma^{2}(z-t)^{2}\right) f_{2}\left(\left(\boldsymbol{\beta}+\frac{1}{2} \boldsymbol{b}\right)^{2}+\gamma^{2}(z+t)^{2}\right),
$$

which depends on $b, s$ as well as the explicit coordinates.

The classical source problem is exactly solvable [8]. The fundamental quantity is the on mass shell Fourier transform of the source

$$
\rho_{k}=\int \mathrm{d}^{3} x \mathrm{~d} t \mathrm{e}^{i k \cdot x-i \sqrt{k^{2}+\mu^{2} t}} \rho(x, t),
$$

where $\mu$ is the mass of the produced object, which we call a "cluster". The $S$-matrix for the classical source problem is 


$$
S=\exp i \int \frac{\mathrm{d}^{3} k}{\sqrt{k^{2}+\mu^{2}}}\left[\rho_{k} \varphi_{k}^{+}+\rho_{k}^{*} \varphi_{k}\right]
$$

where $\varphi_{k}^{+}, \varphi_{k}$ are the creation and destruction operators of a cluster. This expression explicitly exhibits the unitarity of $S$. The $S$-matrix elements are easy to evaluate. We are interested in the transition from zero clusters (only protons) to n clusters (in addition to the protons). For this case we have

$$
\begin{aligned}
S_{n} & \equiv\langle p p+n \varphi ' s|S| p p\rangle \\
& =i^{n} \mathrm{e}^{-\frac{1}{2} \bar{n}} \prod_{j=1}^{n} \rho_{k_{j}},
\end{aligned}
$$

where

$$
\bar{n} \equiv \bar{n}(b, s)=\int \frac{\mathrm{d}^{3} k}{\sqrt{k^{2}+\mu^{2}}}|\rho|_{k}^{2} .
$$

The physical meaning of $\bar{n}$ is discussed below.

We define the amplitude $f$ as

$$
f=1-S
$$

which has $0 \rightarrow n$ matrix elements.

$$
\begin{aligned}
& f_{0}=1-\mathrm{e}^{-\frac{1}{2} \bar{n}}, \\
& f_{n \neq 0}=-i^{n} \mathrm{e}^{-\frac{1}{2} \bar{n}} \prod_{j=1}^{n} \rho_{k_{j}} .
\end{aligned}
$$

We will establish the identification of our elastic amplitude $f_{0}$ [given by eq. (10)] and that of Chou and Yang when we show

$$
\Omega=\frac{1}{2} \bar{n},
$$

where $\Omega$ is the matter density overlap.

In order to understand the model, it is convenient, as we have mentioned, to consider scattering between eigenstates of impact parameter, rather than the experimental conditions of momentum eigenstates. Each cross section is a cross section per unit area in impact parameter space, $\mathrm{d} \sigma / \mathrm{d}^{2} \boldsymbol{b}$, which is dimensionless. Actually, we describe the clusters in momentum space and the protons in impact parameter. The no-recoil assumption implies the conservation of impact parameter. We obtain cross sections by squaring eqs. $(10,11)$ and integrating over phase space. In integrating each cluster over all of phase space, we have counted each configuration $n$ ! times, so we must divide by $n$ ! The cross sections are 


$$
\begin{aligned}
& \frac{\mathrm{d} \sigma_{0}}{\mathrm{~d}^{2} b}=1-2 \mathrm{e}^{-\frac{1}{2} \bar{n}}+\mathrm{e}^{-\bar{n}}, \\
& \frac{\mathrm{d} \sigma_{n \neq 0}}{\mathrm{~d}^{2} b}=\mathrm{e}^{-\bar{n}} \bar{n}^{n} / n !
\end{aligned}
$$

At this point, unitarity for elastic scattering is easily verified. By summing eqs. (13) and (14) we find

$$
\begin{aligned}
& \frac{\mathrm{d} \sigma_{\mathrm{T}}}{\mathrm{d}^{2} \boldsymbol{b}}=1-2 \mathrm{e}^{-\frac{1}{2} \bar{n}}+\sum_{0}^{\infty} \mathrm{e}^{-\bar{n}} \bar{n}^{n} / n ! \\
& \quad=2-2 \mathrm{e}^{-\frac{1}{2} \bar{n}}=2 f_{0} .
\end{aligned}
$$

The average multiplicity at fixed $b$ can be calculated from eq. (14). One sees that for $\mathbf{n} \neq 0$ the multiplicities follow a Poisson distribution with mean $\bar{n}$. This does not imply a Poisson distribution after averaging over the impact parameter, but instead implies a broader distribution. The multiplicity times cross section is

$$
\langle n\rangle \frac{\mathrm{d} \sigma}{\mathrm{d}^{2} b}=\sum_{n \mathrm{e}^{-\bar{n}} \bar{n}^{n} / n !=\bar{n}}
$$

The value of $\langle n\rangle$ depends on the definition of $\sigma$ in eq. (16). We distinguish five interesting cases.

(i) The average multiplicity at fixed $\boldsymbol{b}$, irrespective of whether any interaction occured, but counting each incoming particle equally. For this case we make $\mathrm{d} \sigma=\mathrm{d}^{2} b$ and

$$
\langle n\rangle_{b}=\bar{n} ; \quad \text { (all incident particles). }
$$

(ii) Given that some interaction occurred, $\mathrm{d} \sigma=\mathrm{d} \sigma_{\mathrm{T}}$,

$$
\langle n\rangle_{b}=\bar{n} /\left(2-2 \mathrm{e}^{-\frac{1}{2} \bar{n}}\right) ; \quad\left(\sigma_{\mathrm{T}} \text { normalization }\right) .
$$

(iii) Given that an inelastic interaction occurred.

$$
\langle n\rangle_{b}=\bar{n} /\left(1-\mathrm{e}^{-\bar{n}}\right) ; \quad\left(\sigma_{\text {inel }} \text { normalization }\right),
$$

(iv) and (v) Integrating over $\boldsymbol{b}$,

$$
\langle n\rangle \sigma=2 \pi \int b \mathrm{~d} b \bar{n}(b, s),
$$


where $\sigma=\sigma_{\mathrm{T}}$ or $\sigma_{\text {inel }}$ depending on the normalization chosen. The last of these choices corresponds to the experimentally reported $\langle n\rangle$, minus the two protons and divided by the average number of decay products of the clusters, which we call $\nu$. Therefore, we will use

$$
\langle n\rangle=2+\left(\nu / \sigma_{\text {inel }}\right) 2 \pi \int b \mathrm{~d} b \bar{n}(b, s) .
$$

Eqs. (16)-(21), especially eq. (17) describe the physical interpretation of $\bar{n}$.

We will now give a simple argument to show that the clusters produced have vacuum quantum numbers. Suppose, for example, that a cluster did have non-zero charge; then this charge necessarily comes from one of the two initial nucleons. (The interesting case is one neutron and one proton). Eq. (8) shows that the quantity $\bar{n}$ is the integral over phase space of the square of the source $\rho_{k}$, which corresponds to the emission and subsequent absorption of a cluster. When the cluster is re-absorbed, its charge may be returned to the nucleon from which it was derived, or it may be transferred to the other nucleon. In the latter case, there is a net charge transfer; and thus, $\bar{n}$ makes a significant contribution to charge transfer processes, for example pn $\rightarrow$ np. Since such processes are expected to become small at high energies, we want no charge transfer contribution to $\bar{n}$. Similar arguments can be used for the exchange of other quantum numbers, and we conclude that the produced clusters have vacuum quantum numbers. This conclusion is easily verified by a matrix model similar to the $q$-number model described below.

The identification of our expression with that of Chou and Yang comes from combining eqs. (1), (5) and (8). We make the approximation that the $z$ and $t$ dependences of the wave functions can be factored out, and that the longitudinal momenta are large, compared to the transverse momenta. We discuss this approximation below. With these approximations, the integrations over longitudinal and 4th component coordinates simply give a function of energy. The transverse coordinates give

$$
\begin{aligned}
\bar{n}= & \mathrm{fcn}(s) \int \mathrm{d}^{2} k_{\perp}\left|\rho\left(k_{\perp}\right)\right|^{2} \\
& =\mathrm{fcn}(s) \int \mathrm{d}^{2} k_{\perp} \int \mathrm{d}^{2} x_{\perp} \psi_{\perp}\left(x_{\perp}\right) \psi_{2}\left(x_{\perp}\right) \mathrm{e}^{i k_{\perp} \cdot x_{\perp}} \\
& \times \int \mathrm{d}^{2} x_{\perp}^{\prime} \psi_{1}^{*}\left(x_{\perp}^{\prime}\right) \psi_{2}^{*}\left(x_{\perp}^{\prime}\right) \mathrm{e}^{-i k_{\perp}^{\prime} \cdot \mathrm{x}_{\perp}^{\prime}} \\
& =\mathrm{fcn}(s) \int \mathrm{d}^{2} x_{\perp}\left|\psi_{1}\left(x_{\perp}\right)\right|^{2}\left|\psi_{2}\left(x_{\perp}\right)\right|^{2},
\end{aligned}
$$

which, with an appropriate relationship between our coupling constant and that of Chou and Yang, establishes eq. (12), by identifying the matter density distribution with the absolute square of the wave function. Thus we have demonstrated that our model agrees with Chou and Yang for elastic scattering, as is to be expected by our adoption of their philosophy.

Of course we should use the more exact expressions, eqs. (4), (5) and (8) rather than the approximation leading to eq. (22). This exact treatment leads to interesting 
consequences, such as the lack of factorization of $\Omega$ into a function of $s$ times a function of $b$. Such a lack of factorization seems to be indicated by the ISR elastic scattering data [9]. However, in this paper we do not concern ourselves with this level of refinement, and we shall consider a factorized model. If the wave function were Gaussian, it would be factorizable. We shall modify the wave function slightly, giving up exact spherical symmetry. We choose

$$
\psi\left(r_{\perp}, r_{\|}\right)=G\left(r_{\perp}^{2}\right) \mathrm{e}^{-r_{\|}^{2} / b_{0}^{2}} .
$$

In order to have approximate spherical symmetry we require

$$
G\left(r_{\perp}^{2}\right) \approx \mathrm{e}^{-r_{\perp}^{2} / b_{0}^{2}},
$$

so that

$$
b_{0}^{2}=\frac{2 \int G^{2}\left(r_{\perp}^{2}\right) r_{\perp}^{2} \mathrm{~d} r_{\perp}^{2}}{\int G^{2}\left(r_{\perp}^{2}\right) \mathrm{d} r_{\perp}^{2}}=2\left\langle r_{\perp}^{2}\right\rangle .
$$

This equation is to be understood as only approximate in the realisitic case that $G$ is far from a Gaussian. In order to complete the factorization we replace $k_{\perp}$ in

$$
\sqrt{k^{2}+\mu^{2}}=\sqrt{k_{\|}^{2}+k_{\perp}^{2}+\mu^{2}}
$$

by its average value, and define $\mu_{\perp}^{2}=\left\langle k_{\perp}^{2}\right\rangle+\mu^{2}$, so $\sqrt{k^{2}+\mu^{2}} \approx \sqrt{k_{\|}^{2}+\mu_{\perp}^{2}}$.

With these approximations we have, for the longitudinal dependence from eq. (4),

$$
\rho_{\|}(z, t)=\mathrm{e}^{-\left(2 \gamma^{2} / b_{0}^{2}\right)\left(z^{2}+t^{2}\right)},
$$

whence, from eq. (5)

$$
\begin{aligned}
\rho_{k_{\|}} & =\frac{\pi b_{0}^{2}}{2 \gamma^{2}} \mathrm{e}^{-\left(b_{0}^{2} / 8 \gamma^{2}\right)\left(k_{\|}^{2}+k_{\|}^{2}+\mu_{\perp}^{2}\right),} \\
& =\frac{\pi b_{0}^{2}}{2 \gamma^{2}} \mathrm{e}^{-b_{0}^{2}\left(k_{\|}^{2}+\frac{1}{2} \mu_{\perp}^{2}\right) / 4 \gamma^{2}} .
\end{aligned}
$$

The factor $1 / \gamma^{2}$ is clearly inconsistent with approximately constant total cross sections. Its origin is the decrease in volume caused by the Lorentz contraction. It can be removed by making the wave functions vectors instead of scalars. (Vector wave functions were also used by Calucci et al. [5]). Because of the lack of experimental spin effects in high energy pp scattering, we shall choose $\psi_{\mu}=P_{\mu} f\left(r^{2}\right)$ instead of involving $\gamma_{\mu}$. The factor $P_{\mu_{1}} P_{\mu_{2}} \propto \gamma^{2}$, and the interesting remaining part of $\rho_{k_{\|}}$is 


$$
\mathrm{e}^{-b_{0}^{2}\left(k_{\|}^{2}+\frac{1}{2} \mu_{\perp}^{2}\right) / 4 \gamma^{2}}=\mathrm{e}^{-b_{0}^{2} M^{2}\left(k_{\|}^{2}+\frac{1}{2} \mu_{\perp}^{2}\right) / s}
$$

[see eq. (3)]. When written in rapidity (for $\left.k_{\|} \gg 0\right)$ this is $\exp \left(-b_{0}^{2} \mu_{\perp}^{2} \mathrm{e}^{2(y-Y)}\right)$ which exhibits a rapidity plateau. The rapidity plateau has its origin in the Lorentz contraction. From eq. [8] we obtain the energy dependence of $\bar{n}$ :

$$
\begin{aligned}
\bar{n} & =(\text { fcn of } b) \times \int_{-\infty}^{\infty} \frac{\mathrm{d} k_{11}}{\sqrt{k_{11}^{2}+\mu_{\perp}^{2}}} \mathrm{e}^{-2 b_{0}^{2} M^{2}\left(k_{11}^{2}+\frac{1}{2} \mu_{\perp}^{2}\right) / s} \\
& =(\text { fcn of } b) \times K_{0}\left(b_{0}^{2} \mu_{\perp}^{2} M^{2} / s\right) .
\end{aligned}
$$

As $s \rightarrow \infty, K_{0}$ (const $\left./ s\right) \rightarrow \ln s$, so that the $K_{0}$ function just measures the length of the rapidity plateau, with the appropriate end effects included. Finally, the $b$ dependence, as shown above, is just as in the work of Chou and Yang, with the form factor $F=G^{2}$. Putting everything together we have

$$
\bar{n}=g^{\prime} \int \mathrm{d}^{2} \boldsymbol{\beta} F_{1}\left(\left(\boldsymbol{\beta}-\frac{1}{2} \boldsymbol{b}\right)^{2}\right) F_{2}\left(\left(\boldsymbol{\beta}+\frac{1}{2} \boldsymbol{b}\right)^{2}\right) K_{0}\left(b_{0}^{2} \mu_{1}^{2} M^{2} / s\right) .
$$

In the case of the Gaussian $G=\exp \left(-r_{\perp}^{2} / b_{0}^{2}\right)$, for example, this works out to

$$
\bar{n} \propto \mathrm{e}^{-b^{2} / b_{0}^{2}} K_{0}\left(b_{0}^{2} M^{2} \mu_{\perp}^{2} / s\right)
$$

It is of theoretical, but not phenomenological, interest to calculate the extreme asymptotic behavior of the cross section and the multiplicity. We have found that the eikonal, $\Omega=\frac{1}{2} \bar{n}$, rises at high energy like $\ln s$. At distances much smaller than a distance $R$, the eikonal will be much larger than unity while at distances much larger than $\mathrm{R}$ the scattering is negligible. Therefore

$$
\left.\begin{array}{c}
\sigma_{\mathrm{el}} \\
\sigma_{\text {inel }} \\
\frac{1}{2} \sigma_{\mathrm{T}}
\end{array}\right\} \longrightarrow \pi R^{2} .
$$

The radius $R$ is determined by the condition $\frac{1}{2} \bar{n}(R, s) \approx 1$. The solution of this equation at extreme s depends on the large b dependence of $\bar{n}$. If $\bar{n}$ is a Gaussian $\exp \left(-b^{2} / b_{0}^{2}\right) \ln s$, this works out to $R^{2}=b_{0}^{2} \ln \ln s$, so that at extreme s:

$$
\sigma_{\mathrm{el}} \approx \sigma_{\mathrm{inel}} \approx \frac{1}{2} \sigma_{\mathrm{T}} \approx \pi b_{0}^{2} \ln \ln s .
$$

In this case

$$
\langle n\rangle \sim \frac{\bar{n}}{\sigma} \sim \frac{\ln s}{\ln \ln s} .
$$


A more reasonable choice for the large b dependence of $\bar{n}$ is $\bar{n} \sim \mathrm{e}^{-2 m_{\pi} b} \ln s$. This leads to $R=\left(1 / 2 m_{\pi}\right) \ln \ln s$, so as $\ln \ln s \rightarrow \infty$,

$$
\begin{aligned}
& \sigma_{\mathrm{el}} \approx \sigma_{\mathrm{inel}} \approx \frac{1}{2} \sigma_{\mathrm{T}} \approx \frac{\pi}{4 m_{\pi}^{2}}(\ln \ln s)^{2}, \\
& \langle n\rangle \sim \ln s /(\ln \ln s)^{2} .
\end{aligned}
$$

For phenomenological purposes, these expressions are useless and we use instead the (approximately correct) energy dependence given by eq. (29), or (completely correctly) by eq. (8).

The inclusive cross section for the process

$$
\mathrm{p}+\mathrm{p} \rightarrow \text { cluster + anything }
$$

is also readily calculable by squaring the amplitude $f_{n}$ given in eq. (11), integrating over phase space, and summing over multiplicities. Since the momentum of the observed cluster is not integrated, phase space is counted only $(n-1)$ ! times. Defining $E_{j}=\sqrt{k_{j}^{2}+\mu^{2}}$,

$$
\begin{gathered}
E_{\mathrm{CL}} \frac{\mathrm{d} \sigma(b)}{\mathrm{d}^{3} k_{\mathrm{CL}} \mathrm{d}^{2} b}=\sum_{n=1}^{\infty} \frac{\mathrm{e}^{-\bar{n}(b)}}{(n-1) !} \prod_{j=1}^{n-1} \int \frac{\mathrm{d}^{3} k_{j}}{E_{j}}\left[\rho_{k_{j}}(b)\right]^{2}\left[\rho_{k_{\mathrm{CL}}}(b)\right]^{2} \\
=\mathrm{e}^{-\bar{n}(b)}\left[\rho_{k_{\mathrm{CL}}}(b)\right]^{2} \sum_{n=1}^{\infty} \frac{(\bar{n})^{n-1}}{(n-1) !}=\left[\rho_{k_{\mathrm{CL}}}(b)\right]^{2}
\end{gathered}
$$

so that

$$
E_{\mathrm{CL}} \frac{\mathrm{d} \sigma}{\mathrm{d}^{3} k_{\mathrm{CL}}}=\int \mathrm{d}^{2} b\left[\rho_{k_{\mathrm{CL}}}(b)\right]^{2} .
$$

The inclusive cross section is thus determined by the square of the classical source density. The absence of an absorptive factor $e^{-\bar{n}}$ in eq. (36) is discussed in ref. [10]. The rapidity plateau and transverse momentum cutoff come from the shape of $\dot{\phi}$. In particular, for Gaussian wave functions $\psi(r)=\exp \left(-r^{2} / b_{0}^{2}\right)$, we find

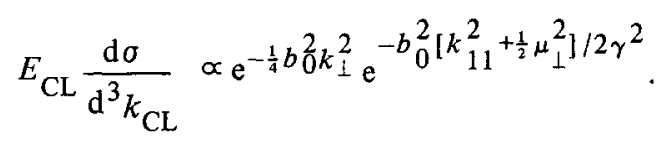

As previously mentioned, the second factor shows a rapidity plateau. Note the Gaussian cutoff in transverse cluster momentum, following from the Gaussian wave functions. The average value of $k_{\perp}$ is given by 


$$
\left\langle k_{\perp}^{2}\right\rangle=\frac{4}{b_{0}^{2}} .
$$

The model we have just finished describing corresponds to the " $c$-number" model of Chou and Yang. In a second paper they describe a " $q$-number" model [3], including diffractive inelastic scattering, whose extension to multiparticle production we now describe.

For this model the wave functions $\psi_{1}$ and $\psi_{2}$ are not simply numbers, but are matrices in a space of states all with the same quantum numbers as the proton. We call one of these states $P^{*}$. The model, in addition to describing $p p \rightarrow p p$ and

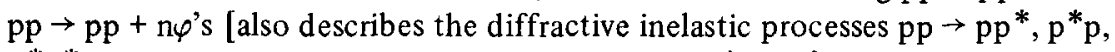
$\mathrm{p}^{*} \mathrm{p} *$ and non-diffractive processes such as $\mathrm{pp} \rightarrow \mathrm{pp} *+n \varphi$ 's.] The development of the model proceeds as above, except that $\rho, \bar{n}$, and the amplitudes become matrices. The most important change is that the zero cluster amplitude $f_{0}$ includes both elastic and diffractive inelastic scattering.

A convenient method for presenting the model is in terms of the eigenstates $|i\rangle$ of $\psi_{1} \psi_{2}$. These eigenstates are in turn eigenstates of $\rho$, of $\bar{n}$, and of diffractive scattering. The probability of a 2 proton state being in a given eigenstate is

$$
P_{i}=|\langle i \mid \mathrm{pp}\rangle|^{2} .
$$

In terms of this eq. ( 8 ) becomes

$$
\langle\mathrm{pp}|\bar{n}| \mathrm{pp}\rangle=\sum_{i}\langle\mathrm{pp} \mid i\rangle \bar{n}_{i}\langle i \mid \mathrm{pp}\rangle=\sum_{i} P_{i} \bar{n}_{i}
$$

so that eq. (21) reads

$$
\langle n\rangle=2 \nu_{L}+\left(\nu / \sigma_{\text {inel }}\right) 2 \pi \int b \mathrm{~d} b \Sigma P_{i} \bar{n}_{i}(b, s),
$$

where we allow the $\mathrm{p}^{*}$ states to decay into $\nu_{L}$ particles on the average. Eq. (10) now has 2 parts,

$$
f_{\mathrm{el}}=\left\langle\mathrm{pp}\left|f_{0}\right| \mathrm{pp}\right\rangle=1-\Sigma P_{i} \mathrm{e}^{-\frac{1}{2} \bar{n}} i,
$$

and the diffractive inelastic part. The elastic cross section is

$$
\frac{\mathrm{d} \sigma_{\mathrm{el}}}{\mathrm{d}^{2} b}=f_{\mathrm{el}}^{2} \text {. }
$$

The diffractive inelastic cross section $\sigma_{\text {diff }}$ is

$$
\begin{aligned}
& \frac{\mathrm{d} \sigma_{\mathrm{diff}}}{\mathrm{d}^{2} b}=\sum_{p^{*} \neq p}\left|\left\langle p^{*} p^{*}\left|f_{0}\right| p p\right\rangle\right|^{2}=\sum_{\text {all }}\left|\left\langle p^{*} p^{*}\left|f_{0}\right| p p\right\rangle\right|^{2}-\frac{\mathrm{d} \sigma_{\mathrm{el}}}{\mathrm{d}^{2} b} \\
& \quad=\left\langle p p\left|f_{0}^{2}\right| p p\right\rangle-\frac{\mathrm{d} \sigma_{\mathrm{el}}}{\mathrm{d}^{2} b}
\end{aligned}
$$


so that

$$
\begin{gathered}
\frac{\mathrm{d} \sigma_{\mathrm{diff}}}{\mathrm{d}^{2} \boldsymbol{b}}=\sum P_{i}\left(1-\mathrm{e}^{-\frac{1}{2} \bar{n}} i\right)^{2}-\frac{\mathrm{d} \sigma_{\mathrm{el}}}{\mathrm{d}^{2} \vec{b}} \\
=\sum_{i<j} P_{i} P_{j}\left[\mathrm{e}^{-\frac{1}{2} \bar{n}_{i}}-\mathrm{e}^{-\frac{1}{2} \bar{n}_{j}}\right]^{2} .
\end{gathered}
$$

Finally, eq. (29) holds for each separate eigenstate.

We could proceed further, decomposing the $n$-cluster cross section into states with p's and $\mathrm{p}^{*}$ 's, etc. Such things are however, not particularly interesting. We will only discuss the consequences of the equations we have presented.

\section{Qualitative results of the model}

Before proceeding to some quantitative comparisons of our model with experimental data, we summarize the qualitative features resulting from the model.

The fundamental interaction is the production of clusters. These clusters must carry isoscalar quantum numbers. The $S$-matrix is exactly unitary. It coincides exactly with the Chou and Yang amplitude for elastic scattering and for diffractive inelastic scattering when that is included in the model. A central quantity is $\bar{n}(b, s)$, which is the average number of resulting clusters at a given energy and impact parameter, irrespective of whether any interaction occurred. This quantity, $\bar{n}(b, s)$, increases at high energy approximately like $\ln s^{*}$. This dependence follows from the available extent of rapidity space; or equivalently from the Lorentz contraction. In the explicit example of Gaussian wave functions [eq. (28)], $\bar{n}$ was proportional to the Bessel function $K_{0}$ (constant/s) which behaves like ln s for large $s$. The $K_{0}$ form is to be preferred to the ln $s$ form even for non-Gaussian wave functions.

The opacity or eikonal $\Omega(b, s)$ is $\frac{1}{2} \bar{n}(b, s)$ [eq. (12)]. Since the opacity increases as a function of $s$, the total and elastic cross sections [which follow from eq. (10)] also rise, In the example of Gaussian wave functions, the asymptotic behavior of the total and elastic cross sections is like $\ln \ln s$ [eq. (32)].

The product of the experimentally observable average cluster multiplicity $\left\langle n_{\mathrm{CL}}\right\rangle$ and the inelastic cross section is proportional to the opacity $\Omega(b, s)$. [See eq. (20)]; hence this product increases like ln $s$. Again, for the case of Gaussian wave functions, one finds that $\left\langle n_{\mathrm{cl}}\right\rangle \rightarrow \ln s / \ln \ln s$, since $\sigma_{\text {inel }}\langle s\rangle \equiv \sigma_{\mathrm{T}}(s)-\sigma_{\mathrm{el}}(s) \rightarrow \ln \ln s$. For wave functions with Yukawa tails the corresponding results are $\sigma_{\mathrm{T}}, \sigma_{\mathrm{el}}$, and $\sigma_{\text {inel }} \rightarrow(\ln \ln s)^{2}$ while $\left\langle n_{\mathrm{cl}}\right\rangle \rightarrow \ln s /(\ln \ln s)^{2}$. These asymptotic statements are of only theoretical interest; the phenomenologically interesting statements are that cross sections and multiplicities are rising in a way predicted by the $t$-dependence (or equivalently the $b$ dependence) of the elastic amplitude.

* The In $s$ factor in $\bar{n}$ was previously noted by Krisch [16], but the resulting rising cross sections were not discussed. 
Qualitatively, $K_{0}$ (constant $/ s$ ) is good for fitting the multiplicity since it rises more slowly at small $s$ than $\ln s$ as does the data. $K_{0}$ is positive for all $s$, while $\ln s$ is negative for small $s$. Inclusive cross sections exhibit a rapidity plateau, have scaling both in the central and end regions, and have a transverse momentum cutoff. The end effects in rapidity are given by the same $K_{0}$ function that appears in $\bar{n}$, as well as by the actual fragmentation (if any) of the incident particles. Typically, the average transverse momentum of a cluster is given by

$$
\left\langle k_{\perp}^{2}\right\rangle=\frac{4}{b_{0}^{2}}
$$

The value of $b_{0}^{2}$ at ISR energies can be estimated from fig. 2 of ref. [11], and it lies in the range $0.045 \leqslant b_{0}^{-2} \leqslant 0.08(\mathrm{GeV} / c)^{2}$. Consequently, $0.18 \leqslant\left\langle k_{\perp}^{2}\right\rangle \leqslant 0.32$ $(\mathrm{GeV} / c)^{2}$. If a cluster were to have a mass $\geq 1 \mathrm{GeV}$, eq. (2) of ref. [12] shows that this range of $\left\langle k_{1}^{2}\right\rangle$ is moderately small, and would be difficult to detect experimentally.

The multiplicity distribution of clusters at fixed impact parameter is Poisson except for the diffractive events (i.e., those with zero clusters). The actual multiplicity distribution is broadened by averaging over impact parameter and by the decay of clusters.

The qualitative features of the model which can be compared to the experimental situation are extremely encouraging. There remains, for the next section, to make a quantitative comparison with relevant data. The most important thing to check is whether the same $\bar{n}$ can give both the eikonal and the multiplicity. Since the cluster size is arbitrary, we must compare not only the magnitudes of the eikenal and multiplicity, but also compare the energy dependence of the multiplicity with the rise of the total cross section and the associated shrinkage of the elastic peak. This is the place where the multiperipheral model is unsatisfactory [1]. We shall now see how the extended Chou-Yang model fares.

\section{Comparison with experimental data}

We have seen that the classical source Chou-Yang model described above is qualitatively consistent with the trends exhibited by the data; e.g., rising total cross sections and multiplicities. In this section we examine whether quantitative agreement is possible. We first consider the $c$-number model with only a single elastic 2 -body channel (case A); subsequently we look at the $q$-number model with 2 and 4 eigenchannels in order to include the possibility of diffraction dissociation (case B). Finally, we discuss some explicit examples with many eigenchannels (case $\mathrm{C}$ ). 
Case A: The relevant formulae are the usual eikonal expressions

$$
\begin{aligned}
& \sigma_{\mathrm{T}}(s)=4 \pi \int_{0}^{\infty} b \mathrm{~d} b f(s, b)=4 \pi \int_{0}^{\infty} b \mathrm{~d} b\left[1-\mathrm{e}^{-\Omega(s, b)}\right], \\
& \sigma_{\mathrm{el}}(s)=2 \pi \int_{0}^{\infty} b \mathrm{~d} b[f(s, b)]^{2}=2 \pi \int_{0}^{\infty} b \mathrm{~d} b\left[1-\mathrm{e}^{-\Omega(s, b)}\right]^{2}, \\
& \sigma_{\text {inel }}(s) \equiv \sigma_{\mathrm{T}}(s)-\sigma_{\mathrm{el}}(s)=2 \pi \int_{0}^{\infty}\left[1-\mathrm{e}^{-2 \Omega(s, b)}\right] b \mathrm{~d} b, \\
& \frac{\mathrm{d} \sigma}{\mathrm{d} t}=\pi[F(s, t)]^{2} ; F(s, t)=\int_{0}^{\infty} b \mathrm{~d} b f(s, b) J_{0}(b \sqrt{-t}),
\end{aligned}
$$

and in addition, from our extension

$$
\begin{aligned}
& \sigma_{n}(s, b)=\mathrm{e}^{-2 \Omega} \frac{(2 \Omega)^{n}}{n !}, \\
& \left\langle n_{\mathrm{cl}}\right\rangle(s) \sigma_{\text {inel }}(s)=4 \pi \int_{0}^{\infty} b \mathrm{~d} b \Omega(s, b), \\
& \left\langle n_{\mathrm{ch}}\right\rangle=\nu_{\mathrm{ch}}\left\langle n_{\mathrm{cl}}\right\rangle+2 .
\end{aligned}
$$

$\left\langle n_{\mathrm{ch}}\right\rangle$ is the average number of charged particles produced and $\nu_{\mathrm{ch}}$ is the number of charged particles per cluster. We have written $\Omega$ instead of $\frac{1}{2} \bar{n}$ is these expressions. The model is fully specified by a choice of matter density functions $F$ and coupling constant $\mathrm{g}$, which determine the eikonal $\Omega(s, b)$ as given (approximately) by eq. (29). [see also eq. (25)].

In keeping with the original Chou-Yang ideas, the opacity is taken to be proportional to the overlap of electromagnetic form factors. For protons, the form factor is well approximated by the dipole formula $G(t)=\left(1-t / \mu^{2}\right)^{-2}$ with $\mu^{2}=$ $0.71(\mathrm{GeV} / \mathrm{c})^{2}$, and the opacity is

$$
\Omega(s, b) \propto \int_{0}^{\infty}(\sqrt{-t}) \mathrm{d}(\sqrt{-t}) G^{2}(t) J_{0}(b \sqrt{-t})=\kappa(s)(\mu b)^{3} K_{3}(\mu b) .
$$

The energy dependence resides solely in the proportionality function $k(s)$. At this point we do not impose the relationship between the $s$ and the $b$ dependence, but instead choose $\kappa(s)$ to give the correct energy dependence of $\sigma_{\mathrm{T}}(s)$. The elastic differential cross section obtained from the opacity of eq. (47) is known to be in 
very good agreement with the corresponding ISR measurements [4]. Since the opacity is now fully determined, Eq. (46) predicts the energy dependence of the charged particle multiplicity in pp collisions. Evaluation of this expression shows that the rise predicted by the model is approximately $5 \%$ over the ISR energy range [900 $\left.(\mathrm{GeV} / c)^{2} \leqslant s \leqslant 2800(\mathrm{GeV} / c)^{2}\right]$; considerably less than the rise of $\simeq 25 \%$ seen in the data. Moreover, the average number of produced clusters is $\approx 1.5$, which makes each cluster have about 8 particles. Thus, the $c$-number model must be rejected on this basis.

The small number of clusters and the failure to get the desired rise in multiplicity can be understood by the smallness of the opacity function. For small $\Omega$, such that $\mathrm{O}\left(\Omega^{2}\right)$ is not substantial, we have

$$
\begin{aligned}
& \sigma_{\text {inel }}(s) \simeq 4 \pi \int \Omega(s, b) b \mathrm{~d} b, \\
& \left\langle n_{\mathrm{cl}}\right\rangle=\frac{4 \pi}{\sigma_{\text {inel }}(s)} \int b \mathrm{~d} b \Omega(s, b) \simeq 1,
\end{aligned}
$$

independent of $s$. Roughly speaking, when $\Omega$ is small, eikonalization is not important, and the energy dependence of the opacity is more or less cancelled by that of the inelastic cross section, leaving an essentially flat value for the number of produced clusters. The situation would be quite different for large opacity functions; in that case the impact parameter amplitude would approximately be a black disc $\sigma_{\text {inel }}(s)$ would have little energy dependence, and the energy dependence of the opacity function would be reflected in $\left\langle n_{\mathrm{cl}}\right\rangle$.

The small number of clusters produced can also be understood in terms of an argument by Goldman and Sivers [13]. Their argument applied to a version of the multiperipheral model rather than to the Chou-Yang model, but can be expressed in terms of our considerations. They draw a connection between the use of vector wave functions and the extremely small value of the multiplicity. Since we are forced to use vector wave functions, we are forced to have a low multiplicity.

Although we have ruled out the $c$-number model, it still is of some interest to impose the condition from eq. (29), that

$$
\kappa(s)=K_{0}\left(\frac{b_{0}^{2} \mu_{\perp}^{2} M^{2}}{s}\right)
$$

to see if this can fit the energy dependence of just the total cross section. As mentioned in sect. 3 , the value of $b_{0}^{-2}$ is between 0.045 and 0.08 . The parameter $\mu_{\perp}=$ cluster transverse mass can be adjusted to fit the rise in $\sigma_{\mathrm{T}}$ in the ISR region. The value is somewhat sensitive to the amount of rise of $\sigma_{\mathrm{T}}$, but throughout the range of reasonable values chosen turns out to be $\mu_{\perp}^{2} b_{0}^{2} \lesssim 1$. Such a low value is impossible since 


$$
\mu_{\perp}^{2} b_{0}^{2}=\mu^{2} b_{0}^{2}+k_{\perp}^{2} b_{0}^{2}>4 m_{\pi}^{2} b_{0}^{2}+k_{\perp}^{2} b_{0}^{2} \approx 1+4=5
$$

by using even the smallest possible value of $b_{0}$ and by using eq. (39). Reasonable values of $\mu_{\perp}$ and $b_{0}$ would give $\mu_{1}^{2} b_{0}^{2} \approx 15$ or larger. Therefore, also for this reason the $c$-number model must be ruled out.

Moreover, in our extension, there is no additional mechanism available for diffractive inelastic scattering. The existence of diffractive inelastic scattering by itself rules out the $c$-number model and forces us to consider the $q$-number model.

Case B: The quantitatively un-successful results of case A naturally lead us to the next more complicated (yet more realistic) case of two 2-body eigenchannels of the eikonal $\Omega$. Thus diffraction dissociation is now incoporated in the model. While it is true that we now have additional freedom, it must be remembered that we must also now explain additional facts pertaining to diffraction, e.g., that the diffractive cross section at ISR energies $\simeq 7 \mathrm{mb}$. The relevant formulae are

$$
\begin{aligned}
& f_{i}(s, b)=1-\mathrm{e}^{-\Omega_{i}(s, b)},(i=1,2), \\
& \sum_{i=1,2} P_{i}=1, \\
& f=\sum_{\mathrm{i}=1,2} P_{i} f_{i}, \\
& \sigma_{\mathrm{diff}}=2 \pi P_{1} P_{2} \int_{0}^{\infty} b \mathrm{~d} b\left[f_{1}(s, b)-f_{2}(s, b)\right]^{2}, \\
& \left\langle n_{\mathrm{cl}}\right\rangle(s) \sigma_{\text {inel }}(s)=4 \pi \int_{0}^{\infty} b \mathrm{~d} b \sum_{i=1,2} P_{i} \Omega_{i}(s, b) .
\end{aligned}
$$

Note that the diffractive cross section involves the difference $f_{1}(s, b)-f_{2}(s, b)$, or $f_{i}-f_{j}$ if there are more than two channels. In order to get a substantial $\sigma_{\text {diff }}, f_{1}$ and $f_{2}$ must necessarily be sufficiently different, which would hold if one of the eikonals $\Omega_{i}$ is considerably bigger than the other. Let us recall [see discussion preceding eq. (47)] that amplitudes with large eikonals are heavily influenced by eikonalization, and therefore give a much larger rise in multiplicity relative to the rise in $\sigma_{\mathrm{T}}$. Thus, considering a $q$-number model is a qualitatively sound step in the right direction.

The association of the eikonals $\Omega_{i}$ with nucleon electromagnetic form factors cannot be made in the $q$-number model. This can be understood as a result of forcing the $\Omega_{i}$ 's apart. Those $\Omega_{i}$ 's which are larger than the $\Omega$ of the $c$-number model are flattened very much by eikonalization, much more than enough to com- 
pensate for the lesser flattening of the small eikonals. This leads to a flat amplitude, similar to $\theta(1 \mathrm{fm}-\mathrm{b})$, which causes a dip in elastic scattering at $-t \approx 0.6(\mathrm{GeV} / c)^{2}$. This dip position occurs if the amount of diffraction dissociation is anywhere near the experimental value of $\approx 7 \mathrm{mb}$. How little diffraction can ruin the fit is illustrated by the work of Elitzur and Lipes [14]. They included only $0.7 \mathrm{mb}$ of diffraction, yet this was sufficient to move the dip from the experimental value of $t=-1.3$ to $\mathrm{t}=-0.9(\mathrm{GeV} / \mathrm{c})^{2}$.

The features of the model we are concerned with depend on $\Omega_{i}(s, b)$ and the probability $P_{1}$. The straightforward connection between the opacities $\Omega_{i}$ and the electromagnetic form factors is now lost. However, in order to describe the elastic differential cross section, instead of fitting experimental data directly, we impose the constraint [as in case $\mathrm{A}$ ] that the elastic amplitude be given by

$$
\begin{aligned}
& f(s, b)=1-\mathrm{e}^{-\kappa(s)(\mu b)^{3} K_{3}(\mu b)}=1-\mathrm{e}^{-\Omega(s, b)} \\
& \quad=P_{1}\left[1-\mathrm{e}^{-\Omega_{1}(s, b)}\right]+\left(1-P_{1}\right)\left[1-\mathrm{e}^{-\Omega_{2}(s, b)}\right] .
\end{aligned}
$$

We shall assume that $\Omega_{1}$ and $\Omega_{2}$ are proportional $\Omega_{1}(s, b)=h \Omega_{2}(s, b)$. This amounts to saying that the opacities corresponding to the elastic scattering of various eigenstates have the same shape. A straightforward generalization to the more physically reasonable case of 4 eigenchannels with $\Omega_{1}=h \Omega_{2}=h \Omega_{3}=h^{2} \Omega_{4}$ can be made. The remaining free parameters and $0 \leqslant P_{1} \leqslant 1$ were varied in an attempt to get $\sigma_{\text {diff }} \simeq 7 \mathrm{mb}$ and a $25 \%$ rise in the average multiplicity. This turned out to be a difficult proposition. The best results were obtained with $h \simeq 20$ and $P_{1} \cong 0.4$ in the 4-channel case. The results were $\sigma_{\text {diff }} \simeq 5 \mathrm{mb}$ and a $20 \%$ rise in $\left\langle n_{\mathrm{cl}}\right\rangle$ over the ISR range. The average number of clusters is approximately 3 . These are not entirely satisfactory to explain the experimental data, and moreover $h=20$ is probably unreasonably large by an order of magnitude. Curves of the eikonal $\Omega_{\mathrm{i}}$ and the diffractive differential cross section $\mathrm{d} \sigma_{\mathrm{diff}} / \mathrm{d}^{2} b$ are shown in figs. 1 and 2. Of course, our choice of constraint (eq. A) quarantees good agreement with total and elastic differential cross sections. In fig. 1, one can see the effects of the unreasonable value of $h$. In fig. 2 , one can see that eikonalization causes diffraction to be peripheral. [15].

Case C: Finally, we shall consider various analytically solvable examples with many eigenchannels.

$$
\text { (i) } f=\sum_{i} P_{i} f_{i} \quad \text { with } f_{i}=A_{i} \mathrm{e}^{-b^{2} / r_{i}^{2}}\left(0<A_{i}<1\right) \text {. }
$$

It readily follows that

$$
\begin{aligned}
& \sigma_{\mathrm{T}}=2 \pi \sum_{i} P_{i} A_{i} r_{i}^{2}, \\
& \sigma_{\mathrm{el}}+\sigma_{\mathrm{diff}}=2 \pi \sum_{i} P_{i} \int b \mathrm{~d} b f_{i}^{2} \leqslant \frac{1}{4} \sigma_{\mathrm{T}} .
\end{aligned}
$$


This last relation immediately shows that since $\sigma_{\mathrm{T}} \sim 40 \mathrm{mb}$ and $\sigma_{\mathrm{el}} \sim 7 \mathrm{mb}$ for $\mathrm{pp}$ scattering at ISR energies, it is impossible to have $\sigma_{\text {diff }}$ greater than approximately $3 \mathrm{mb}$, in contradiction to the measured value of $\simeq 8 \mathrm{mb}$. The constraint $\sigma_{\mathrm{el}}+\sigma_{\mathrm{diff}}$ $\leqslant \frac{1}{4} \sigma_{\mathrm{T}}$ is much stronger than the rigorous bound $\sigma_{\mathrm{el}}+\sigma_{\mathrm{diff}} \leqslant \frac{1}{2} \sigma_{\mathrm{T}}$.

(ii) Another example is to take $N$ amplitudes $f_{i}(s, b)$ to be $\theta$-functions, all of equal probability, and widths determined by making the elastic amplitude $f$ be $A$ $\exp \left(-b^{2} / r_{\mathrm{A}}^{2}\right)$.

Then

$$
f(b)=\sum_{i=1}^{N} P_{i} f_{i}(b) \cong \frac{A}{N} \sum_{i=1}^{N} \theta\left(R_{i}-b\right), \quad \text { with } R_{i}^{2}=r_{A}^{2} \ln \left(\frac{N}{i}\right) .
$$

It readily follows that $\sigma_{\mathrm{T}}(N \rightarrow \infty)=2 \pi A r_{A}^{2}$ and $\sigma_{\mathrm{el}}(N \rightarrow \infty)=\frac{1}{2} \pi A^{2} r_{A}^{2}$.

The sum of the elastic and diffractive cross sections is

$$
\sigma_{\mathrm{el}}+\sigma_{\mathrm{diff}}=2 \pi \sum_{i=1}^{N} P_{i} \int b \mathrm{~d} b f_{i}^{2}=\frac{\pi A^{2} r_{A}^{2}}{N} \sum_{i} \ln \left(\frac{N}{i}\right) .
$$

For $N \rightarrow \infty, \sigma_{\text {el }}+\sigma_{\text {diff }} \rightarrow \pi A^{2} r_{A}^{2}=2 \sigma_{\text {el }}$. Thus, for this example it is possible to have a sufficiently large diffractive cross section. The average multiplicity of clusters is $\left\langle n_{\mathrm{d}}\right\rangle=\left(4 \pi / \sigma_{\text {inel }}\right) \Sigma_{i} P_{i} \int b \mathrm{~d} b \Omega_{i}$ with $\Omega_{i}=-\ln \left(1-f_{i}\right)$. For $N \rightarrow \infty$, we find $\left\langle n_{\mathrm{cl}}\right\rangle=-4 \ln (1-A) / 4(4-A)$. The energy dependence of $A$ is fixed by that of the total cross section. This gives an approximate $10 \%$ rise of $\left\langle n_{\mathrm{cl}}\right\rangle$ over the ISR range.

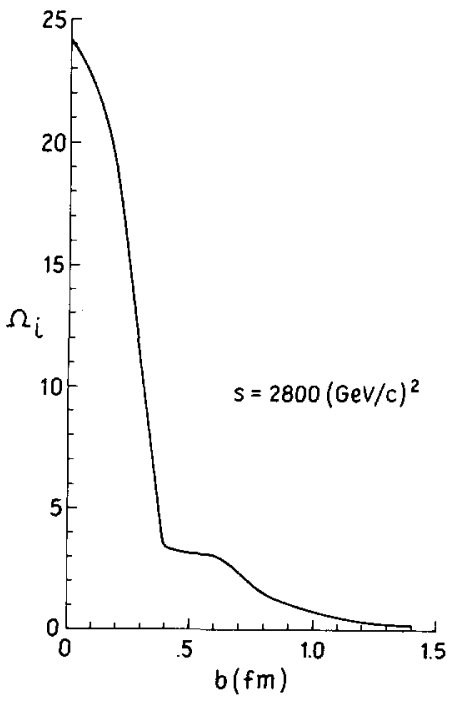

Fig. 1. A plot of the eikonal $\Omega_{i}(s, b)$ versus $b$ for the "best" case described in the text. The kinks are caused by the unreasonable value of the parameter $h$.

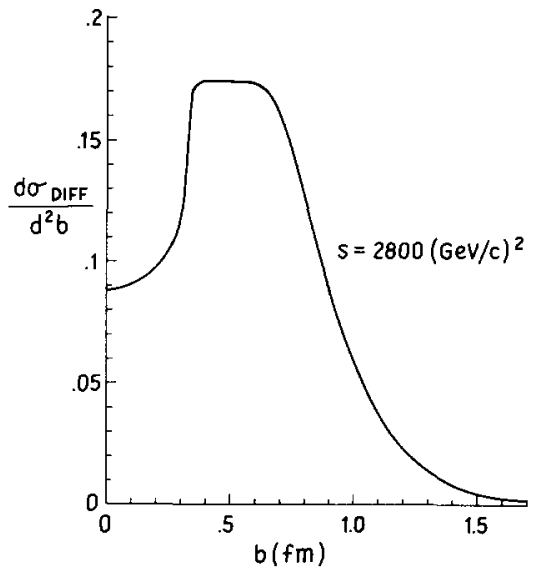

Fig. 2. The diffractive cross section in impact parameter space for the "best" case, showing its peripheral nature as a consequence of eikonalization. 
From these examples, we see that the use of many channels, in itself, does not solve the energy dependence problem.

\section{Conclusions}

We have presented an essentially unique extension of the Chou-Yang model to multiparticle processes. The extension incorporates the ideas of Chou and Yang, and is identical to their model for elastic and diffractive processes, and has an exactly unitary $S$-matrix. The fundamental interaction is the production of clusters with vacuum quantum numbers, which subsequently decay into the observed pions.

There are several major results following directly from the formalism. One is the connection between the multiplicity and the elastic scattering, expressed by the equation that the eikonal is $\frac{1}{2} \bar{n}$. Another is that the extension predicts a multiplicity and total cross section which rise with energy due to Lorentz contractions. This energy dependence is closely related to the momentum transfer dependence. The dependence we use follows from the spherical symmetry of each incoming particle.

The model looks good in terms of most qualitative results. The multiplicity rises approximately like $\ln s$. The total cross section rises. The inclusive distribution has a $P_{\perp}$ cutoff, scales, and exhibits a rapidity plateau because of the Lorentz contractions. The multiplicity distribution is broader than Poisson. Diffractive inelastic scattering, when included, has a peripheral profile in impact parameter.

The existence of diffractive inelastic scattering rules out the $c$-number version of the Chou-Yang model. In our extension, the only source of diffraction is the $q$ number model. In addition, there are two more results of our work which rule out the c-number model: (i) The cluster mass needed falls far below a bound that can be established, and (ii) the multiplicity rises much too slowly.

In the $q$-number model, one is forced to give up the connection with the electromagnetic form factor. If one insists on retaining the electromagnetic form factor, either the amount of diffractive inelastic scattering is extremely small compared to experiment, or the elastic dip is at $t \approx-0.6$ rather than $-1.3(\mathrm{GeV} / c)^{2}$.

The $q$-number model without electromagnetic form factors goes in the right direction toward solving the problems with the $c$-number model, but still falls short of representing the data, even with unreasonable parameter values. The diffractive inelastic cross section tends to less than $6 \mathrm{mb}$ and the multiplicity still rises too slowly.

We have not eliminated all possibility that the $q$-number version can work. We have examined, and rejected, various possibilities. Most importantly, simply including a large number of diffractive channels does not help.

Our principal conclusions are:

(i) An essential assumption of the Chou-Yang model is that a "no-recoil" approximation is good. Without this approximation, the eikonal could not be just a longitudinal integral of a matter density overlap. 
(ii) Given this approximation, the multiparticle extension is unique. We have examined that extension in this paper.

(iii) The extension enjoys many qualitative successes.

(iv) The version of the model without diffraction fails for several reasons.

(v) A slight modification allows us to include diffractive inelastic effects. This version has a great deal of freedom, but yet we were unable to find any case which resembles certain important features of the data.

(vi) In particular, the use of electromagnetic form factors for obtaining the eikonals does not give good results.

(vii) Since the extended Chou-Yang model is unable to provide a basis for a quantitative description of experiment, we seriously question its foundations, especially the no recoil assumption.

We wish to thank G.L. Kane for a critical reading of the manuscript.

\section{References}

[1] F.S. Henyey, Phys. Letters 45B (1973) 469; Nucl. Phys. B78 (1974) 435 and references therein.

[2] T.T. Chou and C.N. Yang, in Proc. of the 2nd Int. Conf. on high-energy physics and nuclear structure, ed. G. Alexander (North-Holland, Amsterdam, 1967); Phys. Rev. 170 (1968) 1591.

[3] T.T. Chou and C.N. Yang, Phys. Rev. 175 (1968) 1832.

[4] F. Hayot and U.P. Sukhatme, Phys. Rev. D10 (1974) 2183;

J.N.J. White, Nucl. Phys. B51 (1973) 23.

[5] G. Calucci, R. Jengo and C. Rebbi, Nuovo Cimento 4A (1971) 330; 6A (1971) 601.

[6] R. Aviv, R.L. Sugar and R. Blankenbecler, Phys. Rev. D5 (1972) 3252.

[7] A.D. Krisch, Phys. Letters 44 (1973) 71 and references therein.

[8] E.M. Henley and W. Thirring, Elementary quantum field theory (McGraw Hill, 1962) ch. 10.

[9] U. Amaldi, in Proc. of the 2nd Int. Conf. on elementary particles, Aix-en-Provence, 1973, J. de Phys. 34 (1973) 1.

[10] M.B. Einhorn and F.S. Henyey, NAL preprint (1974);

J.L. Cardy and G.A. Winbow, Phys. Letters B52 (1974) 95;

C. De Tar, S. Ellis and P.V. Landshoff, Nucl. Phys. B87 (1975) 176.

[11] F.S. Henyey, R. Hong Tuan and G.L. Kane, Nucl. Phys. B 70 (1974) 445.

[12] F. Hayot, F.S. Henyey and M. Le Bellac, Nucl. Phys. B80 (1974) 77.

[13] T. Goldman and D. Sivers, Phys. Letters 48B (1974) 39.

[14] M. Elitzur and R.G. Lipes, Phys. Rev. D7 (1973) 1420.

[15] H.I. Miettinen, in Proc. of the 9th Rencontre de Moriond, Meribel, France, 1974, ed. J. Tran Thanh Van (1974), and references therein.

[16] A.D. Krisch, Phys. Rev. 135 (1964) B 1465. 